\title{
Evidencia Orientada al Paciente: Tratamiento con esteroides de las reagudizaciones de la enfermedad pulmonar obstructiva crónica en personas con diabetes
}

\author{
Steroid treatment for exacerbations of chronic obstructive pulmonary disease in individuals with diabetes
}

Juliana Camoirano

Camoirano J. Tratamiento con esteroides de las reagudizaciones de la enfermedad pulmonar obstructiva crónica en personas con diabetes. Evid Act Pract Ambul. $2016 ; 19(4): 126-127$.

\section{Escenario clínico}

Un paciente de 65 años estaba siendo evaluado en la guardia externa por un cuadro de reagudización de su Enfermedad Pulmonar Obstructiva Crónica (EPOC). Como antecedente de relevancia, presentaba diabetes mellitus tipo II en tratamiento con metformina y medidas higiénico-dietéticas, logrando así un control estable de su enfermedad. Cuando se le informó la necesidad realizar un tratamiento de siete días con corticoides sistémicos como parte del manejo de su cuadro actual, su esposa preguntó si los pacientes con diabetes podían recibir corticoides sin presentar mayores complicaciones.

\section{Pregunta que generó el caso}

En adultos mayores, con diabetes no insulino-dependientes con buen control glucémico habitual y cursando un cuadro de reagudización de EPOC (Población), ¿un ciclo corto de siete días de $40 \mathrm{mg}$ de prednisona vía oral o equivalentes (Intervención) podría generar complicaciones asociadas a la hiperglucemia inducida por corticoides (Resultado/Outcome)?

\section{Estrategia de búsqueda}

Se realizó una búsqueda en MEDLINE empleando como palabras clave "Pulmonary Disease, Chronic Obstructive/complications", "Pulmonary Disease, Chronic Obstructive/drug therapy" y "Diabetes Mellitus/complications". La búsqueda arrojó una única cita relevante para responder la pregunta.

\section{Resumen de la evidencia}

Habib G y col. The impact of corticosteroid treatment on hemoglobin A1C levels among patients with type-2 diabetes with chronic obstructive pulmonary disease exacerbation. Respiratory Medicine (2014) 108, 1641e1646.

Este estudio de casos y control tuvo como objetivo evaluar el efecto del tratamiento con corticoides sistémicos sobre los niveles de la hemoglobina glicosilada $\mathrm{A} 1 \mathrm{C}(\mathrm{HbA} 1 \mathrm{c})$ en pacientes con Diabetes mellitus tipo 2 cursando un cuadro de EPOC reagudizado.

\section{Población}

Casos: Pacientes con diabetes tipo 2 admitidos en el hospital por un cuadro de EPOC reagudizado (grupo 1).

Controles: Pacientes con diabetes tipo 2 con EPOC admitidos por algún otro motivo, diferente a una reagudización de dicha enfermedad de base (grupo 2).

Criterios de exclusión: pacientes con alergia a los corticosteroides; haberlos recibido en forma sistémica, local o tópica durante los tres meses previos; haber comenzado a usar, haber suspendido o cambiado la dosis de esteroides inhalados durante los tres meses previos; haber cambiado durante los tres meses anteriores el tratamiento antidiabético o bien el consumo de carbohidratos. Aquellos que perdieron o ganaron peso en más de un $5 \%$ de su peso original durante los tres meses anteriores o los incapaces por cualquier razón de proporcionar una muestra repetida de sangre para medir $\mathrm{HbA1c} 3$ meses después de la primer extracción.

\section{Mediciones}

25 pacientes fueron reclutados en cada grupo. Sin embargo, el análisis final incluyó 23 pacientes del grupo 1 y 21 del grupo 2 ya que en cuatro pacientes (dos de cada grupo) no se pudo va- lorar los niveles de $\mathrm{HbA} 1 \mathrm{c}$ a los tres meses y además, dos del grupo control recibieron corticoides luego de haber ingresado al estudio.

En ambos grupos se realizó el día de admisión al hospital una extracción de sangre para valorar los niveles de $\mathrm{HbA} 1 \mathrm{c}$, repitiéndose la medición a los tres meses. Además, se recabaron variables demográficas, clínicas y de laboratorio como edad, sexo, índice de masa corporal (IMC), tiempo de desarrollo de diabetes, tratamiento antidiabético y tipo de alimentación. Se evaluó durante la internación y luego de manera ambulatoria, la glucemia, el uso de medicación antidiabética y la dosis de corticoides recibida. Los médicos, enfermeros y nutricionistas a cargo de los pacientes durante su estadía hospitalaria no estuvieron al tanto de la realización del estudio.

\section{Resultados}

Los pacientes que recibieron corticoides presentaron niveles de glucemia significativamente mayores con respecto al grupo control $(p=0.003)$. No hubo diferencias estadísticamente significativas con respecto al aumento o al cambio de la medicación antidiabética o de la reducción en el consumo de carbohidratos entre ambos grupos. Tampoco se observaron diferencias significativas en las mediciones basales y a los tres meses en los niveles de $\mathrm{HbA} 1 \mathrm{c}$ en el grupo $1(\mathrm{p}=0,42)$. Sin embargo, sí se observó una disminución estadísticamente significativa con respecto a los niveles de HbA1c en el grupo $2(p=0,03)$.

\section{Comentarios}

Si bien los autores concluyeron que el uso de corticoides es "seguro" en esta población; los pacientes internados tuvieron, al menos inicialmente, un seguimiento estrecho de sus valores de glucemia con sus respectivas correcciones y ajustes, tanto dietéticos como farmacológicos. Sin embargo, en la práctica no todos los pacientes con EPOC reagudizado presentan criterios de internación, por lo que los resultados de este estudio no serían completamente trasladables a pacientes con un cuadro de manejo ambulatorio, quienes a su vez podrían tener un pobre control glucémico.

Aún si el estudio hubiera arrojado un aumento significativo en el aumento de la $\mathrm{HbA1c}$, caben las siguientes preguntas iel impacto clínico sería de relevancia? ¿la $\mathrm{HbA} 1 \mathrm{c}$ con el tiempo y ya $\sin$ el efecto de los corticoides, no tendería a volver a sus niveles basales? ¿tener durante tres a seis meses valores de $\mathrm{HbA1c}$ mayores a lo esperado genera complicaciones de importancia para el paciente en relación a los riesgos de las complicaciones de la reagudización de EPOC?

Una de las limitaciones de este estudio es la escasa información disponible sobre el grupo empleado como control, por ejemplo el motivo de su internación y las co-intervenciones recibidas para lograr un mejor control glucémico; que podrían haber explicado el mayor descenso de sus niveles de $\mathrm{HbA} 1 \mathrm{c}$ a los tres meses en relación al grupo 1.

Como aporte a la consideración final, no hay que olvidar que la literatura y las guías de práctica clínica apoyan el uso de corticoides sistémicos en las reagudizaciones de EPOC ya que está documentado que reducen el tiempo de recuperación, el riesgo de recaída temprana, la falla terapéutica y el tiempo de internación $n^{1,2,3,4}$

* Servicio de Medicina Familiar y Comunitaria del Hospital Italiano de Buenos Aires. juliana.camoirano@ hospitalitaliano.org.ar 


\section{Conclusiones}

A pesar de que no pudimos encontrar evidencia de alta calidad que responda a la pregunta planteada, consideramos que los riesgos de no dar corticoides a pacientes que están cursando un cuadro EPOC reagudizado superan los beneficios de mantener un estricto control glucémico temporario. Adicionalmente, y considerando las preocupaciones del paciente y su familia podrían reforzarse las indicaciones farmacológicas y no farmacológicas que este viene recibiendo para mantener un buen control glucémico.

Recibido el 02/11/17 y aceptado el 27/01/17.

\section{Referencias:}

1. Ministerio de Salud. Guía de práctica clínica nacional de diagnóstico y tratamiento de la enfermedad pulmonar obstructiva crónica. 2015. p 88. Disponible en: http://www.msal.gob.ar/images/stories/bes/graficos/0000000707cnt-2016-10_guia-practica-clinica-epoc_baja.pdf [Último acceso: diciembre 2016]

2. Alia I y col. Efficacy of corticosteroid therapy in patients with an acute exacerbation of chronic obstructive pulmonary disease receiving ventilatory support. Archives of internal medicine 2011; 171:1939-46.

3. Walters J y col. Different durations of corticosteroid therapy for exacerbations of chronic obstructive pulmonary disease. Cochrane Database Syst Rev 2012.

4. Aaron S y col. Outpatient oral prednisone after emergency treatment of chronic obstructive pulmonary disease. N Engl J Med 2003; 348:2618.

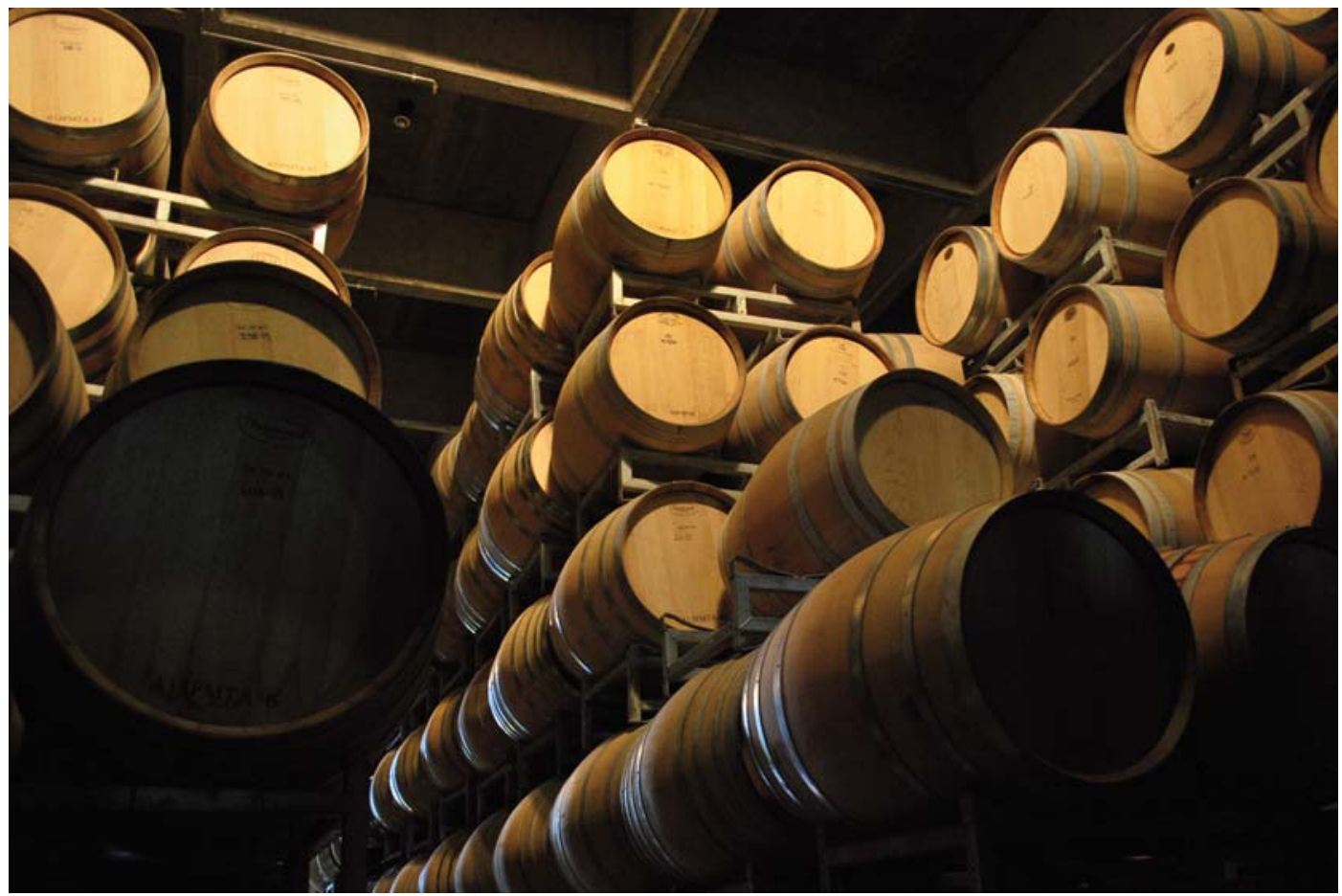

Cortesía: Silvia Spina 